Proyecciones Journal of Mathematics

Vol. 37, $\mathrm{N}^{o}$ 3, pp. 519-533 September 2018.

Universidad Católica del Norte

Antofagasta - Chile

\title{
On the graded classical prime spectrum of a graded module
}

\author{
Khaldoun Al-Zoubi \\ Jordan University of Science and Technology, Jordan \\ and \\ Malik Jaradat \\ Jordan University of Science and Technology, Jordan \\ Received: October 2017. Accepted : March 2018
}

\begin{abstract}
Let $G$ be a group with identity e. Let $R$ be a $G$-graded commutative ring and $M$ a graded $R$-module. In this paper, we introduce and study a new topology on $\mathrm{Cl} . \mathrm{Spec}_{g}(\mathrm{M})$, the collection of all graded classical prime submodules of $M$, called the Zariski-like topology. Then we investigate the relationship between algebraic properties of $M$ and topological properties of $C l$. Spec $_{g}(M)$. Moreover, we study Cl.Spec $(M)$ from point of view of spectral space.
\end{abstract}

Subjclass [2010] : 13A02, 16W50.

Keywords : Graded classical prime submodule, graded classical prime spectrum, Zariski topology. 


\section{Introduction and Preliminaries}

Before we state some results, let us introduce some notations and terminologies. Let $G$ be a group with identity $e$ and $R$ be a commutative ring with identity $1_{R}$. Then $R$ is a $G$-graded ring if there exist additive subgroups $R_{g}$ of $R$ such that $R=\bigoplus_{g \in G} R_{g}$ and $R_{g} R_{h} \subseteq R_{g h}$ for all $g, h \in G$. We denote this by $(R, G)$ (see [8].) The elements of $R_{g}$ are called homogeneous of degree $g$ where the $R_{g}$ 's are additive subgroups of $R$ indexed by the elements $g \in G$. If $x \in R$, then $x$ can be written uniquely as $\sum_{g \in G} x_{g}$, where $x_{g}$ is the component of $x$ in $R_{g}$. Moreover, $h(R)=\bigcup_{g \in G} R_{g}$. Let $I$ be an ideal of $R$. Then $I$ is called a graded ideal of $(R, G)$ if $I=\bigoplus_{g \in G}\left(I \cap R_{g}\right)$. Thus, if $x \in I$, then $x=\sum_{g \in G} x_{g}$ with $x_{g} \in I$. An ideal of a $G$-graded ring need not be $G$-graded (see [8].)

Let $R$ be a $G$-graded ring and $M$ an $R$-module. We say that $M$ is a $G$ graded $R$-module (or graded $R$-module) if there exists a family of subgroups $\left\{M_{g}\right\}_{g \in G}$ of $M$ such that $M=g \in G \bigoplus M_{g}$ (as abelian groups) and $R_{g} M_{h} \subseteq$ $M_{g h}$ for all $g, h \in G$. Here, $R_{g} M_{h}$ denotes the additive subgroup of $M$ consisting of all finite sums of elements $r_{g} s_{h}$ with $r_{g} \in R_{g}$ and $s_{h} \in M_{h}$. Also, we write $h(M)=g \in G \cup M_{g}$ and the elements of $h(M)$ are called homogeneous elements of $M$. Let $M=g \in G \oplus M_{g}$ be a graded $R$-module and $N$ a submodule of $M$. Then $N$ is called a graded submodule of $M$ if $N=g \in G \bigoplus N_{g}$ where $N_{g}=N \cap M_{g}$ for $g \in G$. In this case, $N_{g}$ is called the $g$-component of $N$ (see [8].)

Let $R$ be a $G$-graded ring and $M$ a graded $R$-module. A proper graded ideal $I$ of $R$ is said to be a graded prime ideal if whenever $r s \in I$, we have $r \in I$ or $s \in I$, where $r, s \in h(R)$. The graded radical of $I$, denoted by $\operatorname{Gr}(I)$, is the set of all $x \in R$ such that for each $g \in G$ there exists $n_{g}>0$ with $x^{n_{g}} \in I$. Note that, if $r$ is a homogeneous element, then $r \in \operatorname{Gr}(I)$ if and only if $r^{n} \in I$ for some $n \in \mathbf{N}$. Let $\operatorname{Spec}_{g}(R)$ denote the set of all graded prime ideals of $R$ (see [11].)

A proper graded submodule $N$ of $M$ is said to be a graded prime submodule if whenever $r \in h(R)$ and $m \in h(M)$ with $r m \in N$, then either $r \in(N: R M)=\{r \in R: r M \subseteq N\}$ or $m \in N$ (see [2].) It is shown in [2, Proposition 2.7] that if $N$ is a graded prime submodule of $M$, then $P:=\left(N:_{R} M\right)$ is a graded prime ideal of $R$, and $N$ is called graded $P$-prime submodule. Let $\operatorname{Spec}_{g}(M)$ denote the set of all graded prime submodules of $M$. Note that some graded $R$-modules $M$ have no graded prime submodules. We call such graded modules $g$-primeless. The graded radical of a graded submodule $N$ of $M$, denoted by $G r_{M}(N)$, is defined to be the 
intersection of all graded prime submodules of $M$ containing $N$. If $N$ is not contained in any graded prime submodule of $M$, then $\operatorname{Gr}_{M}(N)=M$ (see $[2,9]$.

A proper graded submodule $N$ of $M$ is called a graded classical prime submodule if whenever $r, s \in h(R)$ and $m \in h(M)$ with $r s m \in N$, then either $r m \in N$ or $s m \in N$ (see [1,4].) Of course, every graded prime submodule is a graded classical prime submodule, but the converse is not true in general (see [1], Example 2.3.) Let $C l . \operatorname{Spec}_{g}(M)$ denote the set of all graded classical prime submodules of $M$. Obviously, some graded $R$ modules $M$ have no graded classical prime submodules; such modules are called $g$-Cl.primeless. The graded classical radical of a graded submodule $N$ of a graded $R$-module $M$, denoted by $G r_{M}^{c l}(N)$, is defined to be the intersection of all graded classical prime submodules of $M$ containing $N$. If $N$ is not contained in any graded classical prime submodule of $M$, then $\operatorname{Gr}_{M}^{c l}(N)=M$ (see [4].) We know that $\operatorname{Spec}_{g}(M) \subseteq C l . \operatorname{Spec}_{g}(M)$. As it is mentioned in ([1], Example 2.3), it happens sometimes that this containment is strict. We call $M$ a graded compatible $R$-module if its graded classical prime submodules and graded prime submodules coincide, that is if $\operatorname{Spec}_{g}(M)=C l . \operatorname{Spec}_{g}(M)$. If $R$ is a $G$-graded ring, then every graded classical prime ideal of $R$ is a graded prime ideal. So, if we consider $R$ as a graded $R$-module, it is graded compatible.

Let $R$ be a $G$-graded ring and $M$ a graded $R$-module. For each graded ideal $I$ of $R$, the graded variety of $I$ is the set $V_{R}^{g}(I)=\left\{P \in \operatorname{Spec}_{g}(R) \mid I \subseteq\right.$ $P\}$. Then the set $\left\{V_{R}^{g}(I) \mid I\right.$ is a graded ideal of $\left.R\right\}$ satisfies the axioms for the closed sets of a topology on $\operatorname{Spec}_{g}(R)$, called the Zariski topology on $\operatorname{Spec}_{g}(R)($ see $[7,10]$.

In [3], $\operatorname{Spec}_{g}(M)$ has endowed with quasi-Zariski topology. For each graded submodule $N$ of $M$, let $V_{*}^{g}(N)=\left\{P \in \operatorname{Spec}_{g}(M) \mid N \subseteq P\right\}$. In this case, the set $\zeta_{*}^{g}(M)=\left\{V_{*}^{g}(N) \mid N\right.$ is a graded submodule of $\left.M\right\}$ contains the empty set and $\operatorname{Spec}_{g}(M)$, and it is closed under arbitrary intersections, but it is not necessarily closed under finite unions. The graded $R$-module $M$ is said to be a $g$-Top module if $\zeta_{*}^{g}(M)$ is closed under finite unions. In this case $\zeta_{*}^{g}(M)$ satisfies the axioms for the closed sets of a unique topology $\tau_{*}^{g}$ on $\operatorname{Spec}_{g}(M)$. The topology $\tau_{*}^{g}(M)$ on $\operatorname{Spec}_{g}(M)$ is called the quasi-Zariski topology.

In [4], Cl.Spec ${ }_{g}(M)$ has endowed with quasi-Zariski topology. For each graded submodule $N$ of $M$, let $\mathbf{V}_{*}^{g}(N)=\left\{C \in C l . \operatorname{Spec}_{g}(M) \mid N \subseteq C\right\}$. In this case, the set $\eta_{*}^{g}(M)=\left\{\mathbf{V}_{*}^{g}(N) \mid N\right.$ is a graded submodule of $\left.M\right\}$ contains the empty set and $C l . \operatorname{Spec}_{g}(M)$, and it is closed under arbitrary 
intersections, but it is not necessarily closed under finite unions. The graded $R$-module $M$ is said to be a $g$-Cl.Top module module if $\eta_{*}^{g}(M)$ is closed under finite unions. In this case $\eta_{*}^{g}(M)$ satisfies the axioms for the closed sets of a unique topology $\varrho_{*}^{g}$ on $C l . S p e c_{g}(M)$. In this case, the topology $\varrho_{*}^{g}(M)$ on $C l . \operatorname{Spec}_{g}(M)$ is called the quasi-Zariski topology.

In this article, we introduce and study a new topology on $C l . \operatorname{Spec}_{g}(M)$, called the Zariski-like topology, which generalizes the Zariski topology of graded rings to graded modules. Let $R$ be a $G$-graded ring and $M$ a graded $R$-module. For each graded submodule $N$ of $M$, we define $\mathbf{U}_{*}^{g}(N)=$ Cl.Spec $g(M)-\mathbf{V}_{*}^{g}(N)$ and put $\mathbf{B}^{\text {cl }}(M)=\left\{\mathbf{U}_{*}^{g}(N): N\right.$ is a graded submodule of $M$ \}. Then we define $\tau_{g}^{c l}(M)$ to be the topology on $C l . \operatorname{Spec}_{g}(M)$ by the sub-basis $\mathbf{B}^{c l}(M)$. In fact $\tau_{g}^{c l}(M)$ to be the collection $U$ of all unions of finite intersections of elements of $\mathbf{B}^{c l}(M)$. We call this topology the Zariskilike topology of $M$.

If $N$ is a graded submodule (respectively proper submodule) of a graded module $M$ we write $N \leq_{g} M$ (respectively $\left.N_{g} M\right)$.

\section{Topology on $C l . \operatorname{Spec}_{g}(M)$}

Let $R$ be a $G$-graded ring and $M$ a graded $R$-module. A graded submodule $C$ of $M$ will be called a graded maximal classical prime if $C$ is a graded classical prime submodule of $M$ and there is no graded classical prime submodule $P$ of $M$ such that $C \subset P$. Let $C l . \operatorname{Spec}_{g}(M)$ be endowed with the Zariski-like topology. For each subset $Y$ of $C l . \operatorname{Spec}_{g}(M)$, We will denote the closure of $Y$ in $C l$. Spec $_{g}(M)$ by $c l(Y)$.

Lemma 2.1. Let $R$ be a $G$-graded ring and $M$ a graded $R$-module.

i) If $Y$ is a nonempty subset of $C l . \operatorname{Spec}_{g}(M)$, then $c l(Y)=\bigcup_{C \in Y} \mathbf{V}_{*}^{g}(C)$.

ii) If $Y$ is a closed subset of $C l . \operatorname{Spec}_{g}(M)$, then $Y=\bigcup_{C \in Y} \mathbf{V}_{*}^{g}(C)$.

\section{Proof.}

i) Clearly, $c l(Y) \subseteq \bigcup_{C \in Y} \mathbf{V}_{*}^{g}(C)$. Let $S$ be a closed subset of $C l . S p e c_{g}(M)$ containing $Y$. Thus, $S=\bigcap_{i \in I}\left(\bigcup_{j=1}^{n_{i}} \mathbf{V}_{*}^{g}\left(N_{i j}\right)\right)$, for some $N_{i j} \leq_{g} M$, $i \in I$ and $n_{i} \in \mathbf{N}$. Let $P \in \bigcup_{C \in Y} \mathbf{V}_{*}^{g}(C)$. Then, there exists $C_{0} \in Y$ 
such that $P \in \mathbf{V}_{*}^{g}\left(C_{0}\right)$ and so $C_{0} \subseteq P$. Since $C_{0} \in S$, then for each $i \in I$ there exists $j, 1 \leq j \leq n_{i}$, such that $N_{i j} \subseteq C_{0}$, and hence $N_{i j} \subseteq C_{0} \subseteq P$. It follows that $P \in S$. Therefore, $\bigcup_{C \in Y} \mathbf{V}_{*}^{g}(C) \subseteq S$.

ii) Clearly $Y \subseteq \bigcup_{C \in Y} \mathbf{V}_{*}^{g}(C)$. For each $C \in Y$ we have $\mathbf{V}_{*}^{g}(C)=\operatorname{cl}(\{C\}) \subseteq$ $\operatorname{cl}(Y)=Y$ by part(i). Hence $\bigcup_{C \in Y} \mathbf{V}_{*}^{g}(C) \subseteq Y$. Therefore, $Y=$ $\bigcup_{C \in Y} \mathbf{V}_{*}^{g}(C)$.

Now the above lemma immediately yields the following result.

Corollary 2.2. Let $R$ be a $G$-graded ring and $M$ a graded $R$-module. Then.

1. $c l(\{C\})=\mathbf{V}_{*}^{g}(C)$, for all $C \in C l . \operatorname{Spec}_{g}(M)$.

2. $Q \in \operatorname{cl}(\{C\})$ if and only if $C \subseteq Q$ if and only if $\mathbf{V}_{*}^{g}(Q) \subseteq \mathbf{V}_{*}^{g}(C)$.

3. The set $\{C\}$ is a closed in $C l$. Spec $_{g}(M)$ if and only if $C$ is a graded maximal classical prime submodule of $M$.

The following theorem shows that for any graded $R$-module $M, C l . \operatorname{Spec}_{g}(M)$ is always a $T_{0}$-space.

Theorem 2.3. Let $R$ be a $G$-graded ring and $M$ a graded $R$-module. Then, $C l$. Spec $_{g}(M)$ is a $T_{0}$-space.

Proof. Let $C_{1}, C_{2} \in C l . \operatorname{Spec}_{g}(M)$. By Corollary 2.2, $\operatorname{cl}\left(\left\{C_{1}\right\}\right)=$ $\operatorname{cl}\left(\left\{C_{2}\right\}\right)$ if and only if $\mathbf{V}_{*}^{g}\left(C_{1}\right)=\mathbf{V}_{*}^{g}\left(C_{2}\right)$ if and only if $C_{1}=C_{2}$.

Now, by the fact that a topological space is a $T_{0}$-space if and only if the closures of distinct points are distinct, we conclude that for any graded $R$-module $M, C l . \operatorname{Spec}_{g}(M)$ is a $T_{0}$-space.

Let $R$ be a $G$-graded ring and $M$ a graded $R$-module. Let every graded classical prime submodule of $M$ is contained in a graded maximal classical prime submodule. We define, by transfinite induction, sets $X_{\alpha}$ of graded classical prime submodule of $M$. To start, let $X_{-1}$ be the empty set. Next, consider an ordinal $\alpha \geq 0$; if $X_{\beta}$ has been defined for all ordinals $\beta<\alpha$, then let $X_{\alpha}$ be the set of those graded classical prime submodules $C$ in $M$ such 
that all graded classical prime submodules proper containing $C$ belong to $\cup_{\beta<\alpha} X_{\beta}$. In particular, $X_{0}$ is the set of graded maximal classical prime submodules of $M$. If some $X_{\gamma}$ contains all graded classical prime submodules of $M$, then we say that $\operatorname{dim}_{g}^{c l}(M)$ exists, and we set $\operatorname{dim}_{g}^{c l}(M)$-the graded classical prime dimension of $M$ to be to the smallest such $\gamma$. We write $\operatorname{dim}_{g}^{c l}(M)=\gamma$ as an abbreviation for the statement that $\operatorname{dim}_{g}^{c l}(M)$ exists and equal $\gamma$. In fact, if $\operatorname{dim}_{g}^{c l}(M)=\gamma<\infty$, then $\operatorname{dim}_{g}^{c l}(M)=\sup \{h t(C) \mid C$ is graded classical prime submodule of $\mathrm{M}\}$. Where $h t(C)$ is the greatest non-negative integer $n$ such that there exists a chain of graded classical prime submodules of $M, C_{0} \subset C_{1} \subset \ldots \ldots \ldots \subset C_{n}=C$, and $h t(C)=\infty$ if no such $n$ exists.

Let $X$ be a topological space and let $x_{1}$ and $x_{2}$ be two points in $X$. We say that $x_{1}$ and $x_{2}$ can be separated if each lies in an open set which does not contain the other point. $X$ is a $T_{1}$-space if any two distinct points in $X$ can be separated. A topological space $X$ is a $T_{1}$-space if and only if all points of $X$ are closed in $X$, (see [6].)

Theorem 2.4. Let $R$ be a $G$-graded ring and $M$ a graded $R$-module. Then $C l . \operatorname{Spec}_{g}(M)$ is $T_{1}$-space if and only if $\operatorname{dim}_{g}^{c l}(M) \leq 0$.

Proof. First assume that $C l . \operatorname{Spec}_{g}(M)$ is a $T_{1}$-space. If $C l . S p a c_{g}(M)=$ $\phi$, then $\operatorname{dim}_{g}^{c l}(M)=-1$. Also, if $C l . \operatorname{Spac}_{g}(M)$ has one element, clearly $\operatorname{dim}_{g}^{c l}(M)=0$. So we can assume that $C l . \operatorname{Spec}_{g}(M)$ has more than two elements. We show that every graded classical prime submodules of $M$ is a graded maximal classical prime submodule. To show this, let $C_{1} \subseteq$ $C_{2}$, where $C_{1}, C_{2} \in C l . \operatorname{Spec}_{g}(M)$. Since $\left\{C_{1}\right\}$ is a closed set, $\left\{C_{1}\right\}=$ $\bigcap_{i \in I}\left(\bigcup_{j=1}^{n_{i}} \mathbf{V}_{*}^{g}\left(N_{i j}\right)\right)$, Where $N_{i j} \leq_{g} M$ and $I$ is an index set. So for each $i \in I, C_{1} \in \bigcup_{j=1}^{n_{i}} \mathbf{V}_{*}^{g}\left(N_{i j}\right)$ so that there exists $1 \leq t_{i} \leq n_{i}$ such that $C_{1} \in \mathbf{V}_{*}^{g}\left(N_{i t_{i}}\right)$. Since $C_{1} \subseteq C_{2}, C_{2} \in \mathbf{V}_{*}^{g}\left(N_{i t_{i}}\right)$ for all $i \in I$. This implies that $C_{2} \in \bigcup_{j=1}^{n_{i}} \mathbf{V}_{*}^{g}\left(N_{i j}\right)$, for all $i \in I$. Therefore, $C_{2} \in \bigcap_{i \in I}\left(\bigcup_{j=1}^{n_{i}} \mathbf{V}_{*}^{g}\left(N_{i j}\right)\right)=\left\{C_{1}\right\}$ as desired.

Conversely, suppose that $\operatorname{dim}_{g}^{c l}(M) \leq 0$. If $\operatorname{dim}_{g}^{c l}(M)=-1$, then Cl.Spac $(M)=\phi$, and hence it is a $T_{1}$-space. Now let $\operatorname{dim}_{g}^{c l}(M)=0$. Then $C l . \operatorname{Spac}_{g}(M) \neq \phi$ and for every graded classical prime submodule of 
$M$ is a graded maximal classical prime submodule. Hence for each graded classical prime submodule $C$ of $M, \mathbf{V}_{*}^{g}(C)=\{C\}$, and so $\{C\}$ is a closed set in $C l . \operatorname{Spac}_{g}(M)$. Hence $C l . \operatorname{Spac}_{g}(M)$ is a $T_{1}$-space.

The cofinite topology is a topology which can be defined on every set $X$. It has precisely the empty set and all cofinite subsets of $X$ as open sets. As a consequence, in the cofinite topology, the only closed subset are finite sets, or the whole of $X$ (see [6].)

Now we give a characterization for a graded module $M$ for which $C l . \operatorname{Spec}_{g}(M)$ is the cofinite topology.

Theorem 2.5. Let $R$ be a $G$-graded ring and $M$ a graded $R$-module. Then the following statements are equivalent:

i) Cl.Spec $(M)$ is the confinite topology.

ii) $\operatorname{dim}_{g}^{c l}(M) \leq 0$ and for every graded submodule $N$ of $M$ either $\mathbf{V}_{*}^{g}(N)=$ $C l . \operatorname{Spec}_{g}(M)$ or $\mathbf{V}_{*}^{g}(N)$ is finite.

Proof. $\quad(i) \Rightarrow(i i)$. Assume that $C l . \operatorname{Spec}_{g}(M)$ is the cofinite topology. Since every cofinite topology satisfies the $T_{1}$ axiom, by Theorem $2.4, \operatorname{dim}_{g}^{c l}(M) \leq 0$. Now assume that there exists a graded submodule $N$ of $M$ such that $\left|\mathbf{V}_{*}^{g}(N)\right|=\infty$ and $\mathbf{V}_{*}^{g}(N) \neq C l . \operatorname{Spec}_{g}(M)$. Then $\mathbf{U}_{*}^{g}(N)=C l . \operatorname{Spec}_{g}(M)-\mathbf{V}_{*}^{g}(N)$ is an open set in $C l . \operatorname{Spec}_{g}(M)$ with infinite complement, a contradiction. $(i i) \Rightarrow(i)$. Suppose that $\operatorname{dim}_{g}^{c l}(M) \leq 0$ and for every graded submodule $N$ of $M, \mathbf{V}_{*}^{g}(N)=C l . \operatorname{Spec}_{g}(M)$ or $\mathbf{V}_{*}^{g}(N)$ is finite. Thus every finite union $\bigcup_{j=1}^{n} \mathbf{V}_{*}^{g}\left(N_{j}\right)$ of graded submodules $N_{j}$ $\leq_{g} M$ is also finite or $C l . \operatorname{Spec}_{g}(M)$. Hence any intersection of finite union $\bigcap_{i \in I}\left(\bigcup_{j=1}^{n} \mathbf{V}_{*}^{g}\left(N_{i j}\right)\right)$ of graded submodules $N_{i j} \leq_{g} M$ is finite or $C l . \operatorname{Spec}_{g}(M)$. Hence every closed set in $C l . \operatorname{Spec}_{g}(M)$ is either finite or Cl.Spec $(M)$. Therefore Cl.Spec $g(M)$ is the cofinite topology.

Suppose that $X$ is a topological space. Let $x_{1}$ and $x_{2}$ be points in $X$. We say that $x_{1}$ and $x_{2}$ can be separated by neighborhoods if there exists a neighborhood $U$ of $x_{1}$ and neighborhood $V$ of $x_{2}$ such that $U \cap V=$ $\phi . \quad X$ is a $T_{2}$-space if any two distinct points of $X$ can be separated by neighborhoods (see [6].) It is well-known that if $X$ is a finite space, then $X$ is $T_{1}$-space if and only if $X$ is the discrete space (see [6].) Thus we have the following corollary.

Corollary 2.6. Let $R$ be a $G$-graded ring and $M$ a graded $R$-module such that $C l . \operatorname{Spec}_{g}(M)$ is finite. Then the following statements are equivalent: 
i) Cl.Spec $g(M)$ is $T_{2}$-space.

ii) Cl.Spec $g(M)$ is $T_{1}$-space.

iii) Cl.Spec $(M)$ is the cofinite space.

iv) $C l . \operatorname{Spec}_{g}(M)$ is discrete.

v) $\operatorname{dim}_{g}^{c l}(M) \leq 0$.

Theorem 2.7. Let $R$ be a $G$-graded ring and $M$ a graded $R$-module such that $M$ has $A C C$ on intersection of graded classical prime submodules. Then, $C l . \operatorname{Spec}_{g}(M)$ is a quasi-compact space

Proof. Suppose $M$ is a graded $R$-module such that $M$ has $A C C$ on intersection of graded classical prime submodules. Let be a family of open sets covering $C l . \operatorname{Spec}_{g}(M)$, and suppose that no finite subfamily of covers $C l$. Spec $_{g}(M)$. Since $\mathbf{V}_{*}^{g}(0)=C l$. Spec $_{g}(M)$, then we may use the $A C C$ on the intersection of graded classical prime submodules to choose a graded submodule $N$ maximal with respect to the property that no finite subfamily of covers $\mathbf{V}_{*}^{g}(N)$. We claim that $N$ is a graded classical prime submodule of $M$, for if not, then there exist $m_{\lambda} \in h(M)$ and $r_{g}, s_{h} \in h(R)$, such that $r_{g} s_{h} m_{\lambda} \in N, r_{g} m_{\lambda} \notin N$ and $s_{h} m_{\lambda} \notin N$. Thus $N N+R r_{g} m_{\lambda}$ and $N N+R s_{h} m_{\lambda}$. Hence, without loss of generality, there must exist a finite subfamily' of that covers both $\mathbf{V}_{*}^{g}\left(N+R r_{g} m_{\lambda}\right)$ and $\mathbf{V}_{*}^{g}\left(N+R s_{h} m_{\lambda}\right)$. Let $C \in \mathbf{V}_{*}^{g}(N)$. Since $r_{g} s_{h} m_{\lambda} \in N, r_{g} s_{h} m_{\lambda} \in C$ and since $C$ is graded classical prime, $r_{g} m_{\lambda} \in C$ or $s_{h} m_{\lambda} \in C$. Thus either $C \in \mathbf{V}_{*}^{g}\left(N+R r_{g} m_{\lambda}\right)$ or $C \in$ $\mathbf{V}_{*}^{g}\left(N+R s_{h} m_{\lambda}\right)$, and hence $\mathbf{V}_{*}^{g}(N) \subseteq \mathbf{V}_{*}^{g}\left(N+R r_{g} m_{\lambda}\right) \cup \mathbf{V}_{*}^{g}\left(N+R s_{h} m_{\lambda}\right)$. Thus, $\mathbf{V}_{*}^{g}(N)$ is covered with the finite subfamily ', a contradiction. Therefore, $N$ is a graded classical prime submodule of $M$.

Now, choose $W \in$ such that $N \in W$. Hence $N$ must have a neighborhood $\bigcap_{i=1}^{n} \mathbf{U}_{*}^{g}\left(P_{i}\right)$, for some graded submodule $P_{i}$ of $M$ and $n \in \mathbf{N}$, such that $\bigcap_{i=1}^{n} \mathbf{U}_{*}^{g}\left(P_{i}\right) \subseteq W$. We claim that for each $i(1 \leq i \leq n)$, $N \in \mathbf{U}_{*}^{g}\left(P_{i}+N\right) \subseteq \mathbf{U}_{*}^{g}\left(P_{i}\right)$. To see this, assume that $C \in \mathbf{U}_{*}^{g}\left(P_{i}+N\right)$, i.e., $P_{i}+N C$. So $P_{i} C$, i.e., $C \in \mathbf{U}_{*}^{g}\left(P_{i}\right)$. On the other hand, $N \in \mathbf{U}_{*}^{g}\left(P_{i}\right)$, i.e., $P_{i} N$. Therefore, $P_{i}+N C$, i.e., $C \in \mathbf{U}_{*}^{g}\left(P_{i}+N\right)$. Consequently, $N \in \bigcap_{i=1}^{n} \mathbf{U}_{*}^{g}\left(P_{i}+N\right) \subseteq \bigcap_{i=1}^{n} \mathbf{U}_{*}^{g}\left(P_{i}\right) \subseteq W$. 
Hence $\bigcap_{i=1}^{n} \mathbf{U}_{*}^{g}\left(P_{i}^{\prime}\right)$, where $P_{i}^{\prime}:=P_{i}+N$, is a neighborhood of $N$ such that $\bigcap_{i=1}^{n} \mathbf{U}_{*}^{g}\left(P_{i}^{\prime}\right) \subseteq W$. Since for each $i(1 \leq i \leq n)$, then $N P_{i}^{\prime}, \mathbf{V}_{*}^{g}\left(P_{i}^{\prime}\right)$ can be covered by some finite subfamily ${ }_{i}^{\prime}$ of . But, $\mathbf{V}_{*}^{g}(N) \backslash\left[\bigcup_{i=1}^{n} \mathbf{V}_{*}^{g}\left(P_{i}^{\prime}\right)\right]=$ $\mathbf{V}_{*}^{g}(N) \backslash\left[\bigcap_{i=1}^{n} \mathbf{U}_{*}^{g}\left(P_{i}^{\prime}\right)\right]^{c}=\left[\bigcap_{i=1}^{n} \mathbf{U}_{*}^{g}\left(P_{i}^{\prime}\right)\right] \cap \mathbf{V}_{*}^{g}(N) \subseteq W$, and so $\mathbf{V}_{*}^{g}(N)$ can be covered by ${ }_{1}^{\prime} \bigcup_{2}^{\prime} \cup \ldots \bigcup_{n}^{\prime} \bigcup\{W\}$, contrary to our choice of $N$. Thus, there must exist a finite subfamily of which covers $C l . \operatorname{Spec}_{g}(M)$. Therefore, Cl.Spec $g(M)$ is a quasi-compact space.

\section{Graded modules whose Zariski-like topologies are spectral spaces}

A topological space $X$ is called irreducible if $X \neq \phi$ and every finite intersection of non-empty open sets of $X$ is non-empty. A (non-empty) subset $Y$ of a topology space $X$ is called an irreducible set if the subspace $Y$ of $X$ is irreducible, equivalently if $Y_{1}$ and $Y_{2}$ are closed subset of $X$ and satisfy $Y \subseteq Y_{1} \cup Y_{2}$, then $Y \subseteq Y_{1}$ or $Y \subseteq Y_{2}$ (see [6].)

Let $Y$ be a closed subset of a topological space. An element $y \in Y$ is called a generic point of $Y$ if $Y=c l(\{y\})$. Note that a generic point of the irreducible closed subset $Y$ of a topological space is unique if the topological space is a $T_{0}$-space (see [5].)

A spectral space is a topological space homomorphic to the prime spectrum of a commutative ring equipped with the Zariski topology. Spectral spaces have been characterized by Hochster [5] as the topological space $W$ which satisfy the following conditions:

i) $W$ is a $T_{0}$-space.

ii) $W$ is quasi-compact.

iii) the quasi-compact open subsets of $W$ are closed under finite intersections and form an open basis.

iv) each irreducible closed subset of $W$ has a generic point.

Let $M$ be a $G$-graded $R$-Module and $Y$ a subset of $C l . S p e c_{g}(M)$. We will denote $\bigcap_{C \in Y} C$ by $\Im(Y)$ (note that if $Y=\phi$, then $\Im(Y)=M$ ).

Lemma 3.1. Let $R$ be a $G$-graded ring and $M$ a graded $R$-module. Then for each $C \in C l . \operatorname{Spec}_{g}(M), \mathbf{V}_{*}^{g}(C)$ is irreducible. 
Proof. Suppose that $\mathbf{V}_{*}^{g}(C) \subseteq Y_{1} \cup Y_{2}$, where $Y_{1}$ and $Y_{2}$ are closed sets. Since $C \in \mathbf{V}_{*}^{g}(C)$, either $C \in Y_{1}$ or $C \in Y_{2}$. Without loss of generality we can assume that $C \in Y_{1}$. We have $Y_{1}=\bigcap_{i \in I}\left(\bigcup_{j=1}^{n_{i}} \mathbf{V}_{*}^{g}\left(N_{i j}\right)\right)$, for some $I$, $n_{i}(i \in I)$, and $N_{i j} \leq_{g} M$. Thus $C \in \bigcup_{j=1}^{n_{i}} \mathbf{V}_{*}^{g}\left(N_{i j}\right)$, for all $i \in I$. It follows that $\mathbf{V}_{*}^{g}(C) \subseteq \bigcup_{j=1}^{n_{i}} \mathbf{V}_{*}^{g}\left(N_{i j}\right)$, for all $i \in I$. Thus $\mathbf{V}_{*}^{g}(C) \subseteq Y_{1}$. Therefore $\mathbf{V}_{*}^{g}(C)$ is irreducible.

Theorem 3.2. Let $R$ be a $G$-graded ring, $M$ a graded $R$-module and $Y \subseteq$ $C l . \operatorname{Spec}_{g}(M)$.

i) If $Y$ is irreducible, then $\Im(Y)$ is a graded classical prime submodule.

ii) If $\Im(Y)$ is a graded classical prime submodule and $\Im(Y) \in \operatorname{cl}(Y)$, then $Y$ is irreducible.

Proof. (i) Assume that $Y$ is an irreducible subset of $C l . S p e c_{g}(M)$. Clearly, $\Im(Y)=\bigcap_{C \in Y} C_{g} M$ and $Y \subseteq \mathbf{V}_{*}^{g}(\Im(Y))$. Let $I, J$ be graded ideals of $R$ and $N$ be a graded submodule of $M$ such that $I J N \subseteq \Im(Y)$. It is easy to see that $Y \subseteq \mathbf{V}_{*}^{g}(I J N) \subseteq \mathbf{V}_{*}^{g}(I N) \cup \mathbf{V}_{*}^{g}(J N)$. Since $Y$ is irreducible, either $Y \subseteq \mathbf{V}_{*}^{g}(I N)$ or $Y \subseteq \mathbf{V}_{*}^{g}(J N)$. If $Y \subseteq \mathbf{V}_{*}^{g}(I N)$, then $I N \subseteq C$, for all $C \in Y$. Thus $I N \subseteq \Im(Y)$. If $Y \subseteq \mathbf{V}_{*}^{g}(J N)$, then $J N \subseteq C$, for all $C \in Y$. Hence $J N \subseteq \Im(Y)$. Thus by [1, Theorem 2.1.], $\Im(Y)$ is a graded classical prime submodule of $M$. (ii) Assume that $C:=\Im(Y)$ is a graded classical prime submodule of $M$ and $C \in \operatorname{cl}(Y)$. It is easy to see that $\operatorname{cl}(Y)=\mathbf{V}_{*}^{g}(C)$. Now let $Y \subseteq Y_{1} \cup Y_{2}$, where $Y_{1}, Y_{2}$ are closed sets. Then we have $\mathbf{V}_{*}^{g}(C)=\operatorname{cl}(Y) \subseteq Y_{1} \cup Y_{2}$. Since $\mathbf{V}_{*}^{g}(C) \subseteq Y_{1} \cup Y_{2}$ and by Lemma $3.1, \mathbf{V}_{*}^{g}(C)$ is irreducible, $\mathbf{V}_{*}^{g}(C) \subseteq Y_{1}$ or $\mathbf{V}_{*}^{g}(C) \subseteq Y_{2}$. Hence either $Y \subseteq Y_{1}$ or $Y \subseteq Y_{2}$. Thus $Y$ is irreducible.

Corollary 3.3. Let $R$ be a $G$-graded ring, $M$ a graded $R$-module and $N$ a graded submodule of $M$. Then the subset $\mathbf{V}_{*}^{g}(N)$ of $C l . S p e c_{g}(M)$ is irreducible if and only if $G r_{M}^{c l}(N)$ is a graded classical prime submodule. Consequently, Cl.Spec $(M)$ is irreducible if and only if $G_{M}^{c l}(M)$ is a graded classical prime submodule. 
Proof. $\quad(\Rightarrow)$ Let $Y:=\mathbf{V}_{*}^{g}(N)$ be an irreducible subset of $C l . \operatorname{Spec}_{g}(M)$. Then we have $\Im(Y)=G r_{M}^{c l}(N)$ so that $G r_{M}^{c l}(N)$ is a graded classical prime submodule of $M$ by Theorem 3.2(i).

$(\Leftarrow)$ By $[4$, Proposition 3.4(1)], for each graded submodule $N$ of $M$, $\mathbf{V}_{*}^{g}(N)=\mathbf{V}_{*}^{g}\left(G r_{M}^{c l}(N)\right)$. Now let $G r_{M}^{c l}(N)$ is a graded classical prime submodule of $M$. Then $G r_{M}^{c l}(N) \in \mathbf{V}_{*}^{g}(N)$, and hence by Theorem 3.2 (ii), $\mathbf{V}_{*}^{g}(N)$ is irreducible.

Lemma 3.4. Let $R$ be a $G$-graded ring and $M$ a graded $R$-module. Then

i) Every $C \in C l . \operatorname{Spec}_{g}(M)$ is a generic point of the irreducible closed subset $\mathbf{V}_{*}^{g}(C)$.

ii) Every finite irreducible closed subset of $C l . \operatorname{Spec}_{g}(M)$ has a generic point.

\section{Proof.}

i) is clear by Corollary 2.2(i).

ii) Let $Y$ be an irreducible closed subset of $C l \cdot \operatorname{Spec}_{g}(M)$ and $Y=$ $\left\{C_{1}, C_{2}, \ldots, C_{n}\right\}$, where $C_{i} \in C l . \operatorname{Spec}_{g}(M), n \in \mathbf{N}$. By Lemma 2.1(i), $Y=c l(Y)=\mathbf{V}_{*}^{g}\left(C_{1}\right) \cup \mathbf{V}_{*}^{g}\left(C_{2}\right) \cup \ldots \cup \mathbf{V}_{*}^{g}\left(C_{n}\right)$. Since $Y$ is irreducible, $Y=\mathbf{V}_{*}^{g}\left(C_{i}\right)$ for some $i(1 \leq i \leq n)$. Now by $(i), C_{i}$ is a generic point of $Y$.

Theorem 3.5. Let $R$ be a $G$-graded ring and $M$ a graded $R$-module such that $C l . \operatorname{Spec}_{g}(M)$ is finite. Then $C l . \operatorname{Spec}_{g}(M)$ is a spectral space (with the Zariski-like topology). Consequently, for each finite graded $R$-module $M, C l . \operatorname{Spec}_{g}(M)$ is a spectral space.

Proof. Since $C l . \operatorname{Spec}_{g}(M)$ is finite, every subset of $C l . \operatorname{Spec}_{g}(M)$ is quasi-compact. Hence the quasi-compact open sets of $C l . \operatorname{Spec}_{g}(M)$ are closed under finite intersection and form an open basis (note: this basis is $\beta=\left\{\mathbf{U}_{*}^{g}\left(N_{1}\right) \cap \mathbf{U}_{*}^{g}\left(N_{2}\right) \cap \ldots \cap \mathbf{U}_{*}^{g}\left(N_{k}\right): N_{i} \leq_{g} M, 1 \leq i \leq k\right.$, for some $k \in \mathbf{N}\})$. Also by Theorem 2.3, Cl.Spec $(M)$ a $T_{0}$-space. Moreover, every 
irreducible closed subset of $C l . \operatorname{Spec}_{g}(M)$ has a generic point by Lemma 3.4. Therefore $C l . \operatorname{Spec}_{g}(M)$ is a spectral space by Hochster's characterization.

Let $X$ be a topological space. By the patch topology on $X$, we mean the topology which has as a sub-basis for its closed sets the closed sets and compact open sets of the original space. By a patch we mean a set closed in the patch topology. The patch topology associated to a spectral space is compact and $T_{2}$-space (see [5].)

Definition 3.6. Let $R$ be a $G$-graded ring and $M$ a graded $R$-module, and let $P_{*}^{g}(M)$ be the family of all subsets of $C l . \operatorname{Spec}_{g}(M)$ of the form $\mathbf{V}_{*}^{g}(N) \cap \mathbf{U}_{*}^{g}(K)$, where $N, K \leq_{g} M$. Clearly $P_{*}^{g}(M)$ contains both

$C_{\text {Cl.Spec }}(M)$ and $\phi$ because Cl.Spec $g(M)=\mathbf{V}_{*}^{g}(0) \cap \mathbf{U}_{*}^{g}(M)$ and $\phi=$ $\mathbf{V}_{*}^{g}(M) \bigcap \mathbf{U}_{*}^{g}(0)$. Let $T_{*}^{g}(M)$ be the collection of all unions of finite intersections of elements of $P_{*}^{g}(M)$. Then, $T_{*}^{g}(M)$ is a topology on $C l . \operatorname{Spec}_{g}(M)$ and is called the patch-like topology of $M$, in fact, $P_{*}^{g}(M)$ is a sub-basis for the patch-like topology of $M$.

Theorem 3.7. Let $R$ be a $G$-graded ring and $M$ a graded $R$-module. Then, $C l$. Spec $_{g}(M)$ with the patch-like topology is a $T_{2}$-space.

Proof. Suppose distinct points $C_{1}, C_{2} \in C l . \operatorname{Spec}_{g}(M)$. Since $C_{1} \neq$ $C_{2}$, then either $C_{1} C_{2}$ or $C_{2} C_{1}$. Assume that $C_{1} C_{2}$. By Definition 3.6, $P_{1}:=\mathbf{U}_{*}^{g}(M) \cap \mathbf{V}_{*}^{g}\left(C_{1}\right)$ is a patch-like-neighborhood of $C_{1}$ and $P_{2}:=$ $\mathbf{U}_{*}^{g}\left(C_{1}\right) \cap \mathbf{V}_{*}^{g}\left(C_{2}\right)$ is a patch-like-neighborhood of $C_{2}$. Clearly, $\mathbf{U}_{*}^{g}\left(C_{1}\right) \cap \mathbf{V}_{*}^{g}\left(C_{1}\right)=\phi$, and thus $P_{1} \cap P_{2}=\phi$. Therefore, $C l . S p e c_{g}(M)$ is a $T_{2}$-space.

The proof of the next theorem is similar to the proof of Theorem 2.7.

Theorem 3.8. Let $R$ be a $G$-graded ring and $M$ a graded $R$-module such that $M$ has $A C C$ on intersection of graded classical prime submodules. Then $C l . \operatorname{Spec}_{g}(M)$ with the patch-like topology is a compact space.

Theorem 3.9. Let $R$ be a $G$-graded ring and $M$ a graded $R$-module such that $M$ has $A C C$ on intersection of graded classical prime submodules. Then every irreducible closed subset of $C l . \operatorname{Spec}_{g}(M)$ (with the Zariski-like topology) has a generic point. 
Proof. Let $Y$ be an irreducible closed subset of $C l . \operatorname{Spec}_{g}(M)$. By Definition 3.6 for each $C \in Y, \mathbf{V}_{*}^{g}(C)$ is an open subset of $C l . \operatorname{Spec}_{g}(M)$ with the patch-like topology. On the other hand since $Y \subseteq C l . \operatorname{Spec}_{g}(M)$ is closed with the Zariski-like topology, the complement of $Y$ is open by this topology. This yields that the complement of $Y$ is open with the patchlike topology. So $Y \subseteq C l . \operatorname{Spec}_{g}(M)$ is closed with the patch-like topology. Since $C l$. Spec $_{g}(M)$ is a compact space in patch-like topology by Theorem 3.8 and $Y$ is closed in $C l . \operatorname{Spec}_{g}(M)$, we have $Y$ is compact space in patchlike topology. Now $Y=\bigcup_{C \in Y} \mathbf{V}_{*}^{g}(C)$ by Lemma 2.1(ii) and each $\mathbf{V}_{*}^{g}(C)$ is open in patch-like topology. Hence there exists a finite set $Y_{1} \subseteq Y$ such that $Y=\bigcup_{C \in Y_{1}} \mathbf{V}_{*}^{g}(C)$. Since $Y$ is irreducible, $Y=\mathbf{V}_{*}^{g}(C)=\operatorname{cl}(\{C\})$ for some $C \in Y$. Therefore, $C$ is a generic point for $Y$.

We need the following evident lemma

Lemma 3.10. Assume $\tau_{1}$ and $\tau_{2}$ are two topologies on $X$ such that $\tau_{1} \subseteq \tau_{2}$. If $X$ is quasi-compact in $\tau_{2}$, then $X$ is also quasi-compact in $\tau_{1}$.

Theorem 3.11. Let $R$ be a $G$-graded ring and $M$ a graded $R$-module such that $M$ has $A C C$ on intersection of graded classical prime submodules. Then for each $n \in \mathbf{N}$, and graded submodules $N_{i}(1 \leq i \leq n)$ of $M$, $\mathbf{U}_{*}^{g}\left(N_{1}\right) \cap \mathbf{U}_{*}^{g}\left(N_{2}\right) \cap \ldots \cap \mathbf{U}_{*}^{g}\left(N_{n}\right)$ is a quasi-compact subset of Cl.Spec $\operatorname{Sp}_{g}(M)$ with the Zariski-like topology.

Proof. Clearly, for each $n \in \mathbf{N}$, and each graded submodules $N_{i}(1 \leq i \leq$ $n)$ of $M, \mathbf{U}_{*}^{g}\left(N_{1}\right) \cap \mathbf{U}_{*}^{g}\left(N_{2}\right) \cap \ldots \cap \mathbf{U}_{*}^{g}\left(N_{n}\right)$ is a closed set in $C l . \operatorname{Spec}_{g}(M)$ with patch-like topology. By Theorem 3.8, Cl.Spec $(M)$ is a compact space with the patch-like topology and since every closed subset of a compact space is compact, $\mathbf{U}_{*}^{g}\left(N_{1}\right) \cap \mathbf{U}_{*}^{g}\left(N_{2}\right) \cap \ldots \cap \mathbf{U}_{*}^{g}\left(N_{n}\right)$ is compact in $C l . \operatorname{Spec}_{g}(M)$ with patch-like topology and so by Lemma 3.10, it is quasi-compact in $C l . \operatorname{Spec}_{g}(M)$ with the Zariski-like topology.

Corollary 3.12. Let $R$ be a $G$-graded ring and $M$ a graded $R$-module such that $M$ has $A C C$ on intersection of graded classical prime submodules. Then Zariski-like quasi-compact open sets of Cl.Spec $(M)$ are closed under finite intersections. 
Proof. It suffices to show that the intersection $Q=Q_{1} \cap Q_{2}$ of two Zariski-like quasi-compact open sets $Q_{1}$ and $Q_{2}$ of $C l . \operatorname{Spec}_{g}(M)$ is Zariskilike quasi-compact set. Each $Q_{i}, i=1,2$, is a finite union of members of the open base $\beta=\left\{\mathbf{U}_{*}^{\mathbf{g}}\left(\mathbf{N}_{\mathbf{1}}\right) \cap \mathbf{U}_{*}^{\mathbf{g}}\left(\mathbf{N}_{\mathbf{2}}\right) \cap \ldots \cap \mathbf{U}_{*}^{g}\left(N_{n}\right): N_{i} \leq_{g} M, 1 \leq i \leq n\right.$, for some $n \in \mathbf{N}\}$. Hence $Q=\bigcup_{i=1}^{m}\left(\bigcap_{j=1}^{n_{i}} \mathbf{U}_{*}^{g}\left(N_{j}\right)\right)$. Let $\Gamma$ be any open cover of $Q$. So $\Gamma$ also covers each $\bigcap_{j=1}^{n_{i}} \mathbf{U}_{*}^{g}\left(N_{j}\right)$ which is Zariski-like quasi-compact by Theorem 3.11. Thus each $\bigcap_{j=1}^{n_{i}} \mathbf{U}_{*}^{g}\left(N_{j}\right)$ has a finite subcover of $\Gamma$ and so dose $Q$.

Theorem 3.13. Let $R$ be a $G$-graded ring and $M$ a graded $R$-module such that $M$ has $A C C$ on intersection of graded classical prime submodules. Then Cl.Spec $g(M)$ (with the Zariski-like topology) is a spectral space.

Proof. By Theorem 2.3, Cl.Spec $(M)$ is a $T_{0}$-space. Also, by Theorem 3.11., Cl.Spec $(M)$ is quasi-compact and has a basis of quasi-compact open subsets. Moreover, by Corollary 3.12, the family of quasi-compact open subset of $C l . \operatorname{Spec}_{g}(M)$ is closed under finite intersections. Finally, every irreducible closed subset of $C l . \operatorname{Spec}_{g}(M)$ has generic point by Theorem 3.9. Thus $C l . \operatorname{Spec}_{g}(M)$ is spectral space by Hochster's characterization.

\section{References}

[1] K. Al-Zoubi, M. Jaradat and R. Abu-Dawwas, On graded classical prime and graded prime submodules, Bull. Iranian Math. Soc. 41 (1), pp. 217-225, (2015).

[2] S. E. Atani, On graded prime submodules, Chiang Mai. J. Sci., 33 (1), pp. 3-7, (2006).

[3] A. Y. Darani, Topologies on $\operatorname{Spec}_{g}(M)$, Bul. Acad. Stiinte Repub. Mold. Mat. 3 (67), pp. 45-53, (2011).

[4] A. Y. Darani and S. Motmaen, Zariski topology on the spectrum of graded classical prime submodules, Appl. Gen. Topol., 14 (2), pp. 159169, (2013).

[5] M. Hochster, Prime ideal structure in commutative rings, Trans. Amer. Math. Soc., 137, pp. 43-60, (1969). 
[6] J. R. Munkres, Topology, A First Course, Prentice-Hall, Inc. Eaglewood Cliffs, New Jersey, (1975).

[7] R. L. McCasland, M. E. Moore and P. F. Smith, On the spectrum of a module over a commutative ring, Comm. Algebra, 25, pp. 79-103, (1997).

[8] C. Nastasescu, F. Van Oystaeyen, Graded Ring Theory, Mathematical Library 28, North Holand, Amsterdam, (1982).

[9] K. H. Oral, U. Tekir and A. G. Agargun, On graded prime and primary submodules, Turk. J. Math., 35, pp. 159-167, (2011).

[10] M. Refai. On properties of G-spec $(R)$, Sci. Math. Jpn. 4, pp. 491-495, (2001).

[11] M. Refai and K. Al-Zoubi, On graded primary ideals, Turk. J. Math. 28, pp. 217-229, (2004).

\section{Khaldoun Al-Zoubi}

Department of Mathematics and Statistics, Jordan University of Science and Technology, P. O.Box 3030, Irbid 22110, Jordan

e-mail : kfzoubi@just.edu.jo

and

\section{Malik Jaradat}

Department of Mathematics and Statistics, Jordan University of Science and Technology, P. O. Box 3030, Irbid 22110, Jordan

e-mail : malikjaradat84@yahoo.com 